\title{
Study of non-strange dibaryon resonances via coherent double neutral-pion photoproduction on the deuteron
}

Takatsugu Ishikawa*, Hisako Fujimura, Ryo Hashimoto, Qinghua He, Shun Kaida, Manabu Miyabe, Norihito Muramatsu, Hajime Shimizu, Koutaku Suzuki, Yusuke Tschikawa, Hirohito Yamazaki

Research Center for Electron Photon Science (ELPH), Tohoku University, Sendai 982-0826, Japan

\section{Shin'ichi Masumoto}

Department of Physics, University of Tokyo, Tokyo 113-0033, Japann

\section{Hiroki Kanda, Kazushige Maeda}

Department of Physics, Tohoku University, Sendai 980-8578, Japan

\section{Kyoichiro Ozawa}

Institute of Particle and Nuclear Studies, KEK, Tsukuba 305-0801, Japan

\section{for the FOREST collaboration}

E-mail: ishikawa@lns.tohoku.ac.jp

The cross sections was measured for coherent double neutral-pion photoproduction on the deuteron using the tagged photon beams from the 0.9 and $1.2 \mathrm{GeV}$ primary electron beams at the Research Center for Electron Photon Science, Tohoku University, Japan. The four-momenta of the four photons from the two $\pi^{0}$ decays and deuteron are measured with the FOREST detector, and the $\gamma d \rightarrow \pi^{0} \pi^{0} d$ reaction was identified using 6-constraint kinematic fit (energy and threemomentum conservation, and two $\pi^{0}$ masses). The total cross section is found to be $18.4 \pm 9.2 \mathrm{nb}$ for the $d^{*}(2380)$ dibaryon resonance at $W_{\gamma d}=2.37 \mathrm{GeV}$, which is a candidate of the isoscalar $\Delta \Delta$ quasi-bound state $\mathscr{D}_{03}$, in the $\gamma d \rightarrow \pi^{0} \pi^{0} d$ reaction. The measured excitation function is consistent with the existing theoretical calculation for this reaction. At high incident energies, $\pi^{0} d$ invariant-mass distributions show a peak at approximately $2.15 \mathrm{GeV}$, which can be attributed to a $N \Delta$ quasi-bound state $\mathscr{D}_{12}$. In this contribution, the properties (mass, width, and decay angular distribution) of this peak is presented.

XVII International Conference on Hadron Spectroscopy and Structure - Hadron2017

25-29 September, 2017

University of Salamanca, Salamanca, Spain

\footnotetext{
* Speaker.
} 


\section{Introduction}

The search for two-baryon systems (dibaryons) has a long history [1]. The dibaryon is of great interest, which can be a molecule consisting of two baryons or a spatially compact hexaquark object. Recently, the $d^{*}(2380)$ resonance has been observed in the $p n$-collision reactions by the CELSIUS/WASA and WASA-at-COSY collaborations [2, 3, 4, 5]. The resonance with $m=2.37$ $\mathrm{GeV}, \Gamma=0.07 \mathrm{GeV}$, and $I\left(J^{\pi}\right)=0\left(3^{+}\right)$may be attributed to an isoscalar $\Delta \Delta$ quasi-bound state, $\mathscr{D}_{03}$, predicted by Dyson and Xuong [6]. Here, non-strange dibaryons are classified as $\mathscr{D}_{I J}$ with isospin $I$ and spin $J$. The $d^{*}(2380)$ resonance should be observable in electro- and photo-production reactions if it exists. The $\gamma^{*} d \rightarrow \pi^{0} \pi^{0} d$ reaction, where the $d^{*}(2380)$ resonance is produced as an intermediate state in the $s$ channel, is expected to give the charge distribution of $d^{*}(2380)$. It should be noted that no special treatment is required kinematically for the Fermi motion of nucleons, and that the $\pi^{0} \pi^{0} d$ has just the isoscalar $(I=0)$ component alone. As a first step, we have measured the cross sections for the $\gamma d \rightarrow \pi^{0} \pi^{0} d$ reaction.

\section{Experiment}

Ten series of meson photoproduction experiments [7] were carried out using photon beams [8] from 0.9- and 1.20-GeV electrons in a synchrotron [9] at the Research Center for Electron Photon Science (ELPH), Tohoku University, Japan. Bremsstrahlung photons were produced from the circulating electrons by inserting a carbon wire. The energy of each photon was determined detecting the corresponding scattered electron, and the tagging-energy range was $0.57-0.89(0.75-1.15) \mathrm{GeV}$ for 0.9 (1.20) $\mathrm{GeV}$ primary electrons. The target used in the experiments was liquid deuterium with a thickness of $45.9 \mathrm{~mm}$. All the final-state particles in the $\gamma d \rightarrow \pi^{0} \pi^{0} d$ reaction were measured using an electromagnetic (EM) calorimeter complex, FOREST [10]. The solid angle of FOREST was approximately $88 \%$ in total.

\section{Analysis}

Events detected in the final state containing four neutral particles and a charged particle were selected. Each neutral pion in the $\gamma d \rightarrow \pi^{0} \pi^{0} d$ reaction was identified via its decay into $\gamma \gamma$. The time difference between every two neutral clusters of four was required to be less than $3 \sigma_{t}$, where $\sigma_{t}$ denotes the time resolution for the difference depending on the modules and their measured energies for the two clusters. Deuterons in the final state were detected with the forward hodoscope. The time delay from the average time response between the four neutral clusters was required to be larger than $1 \mathrm{~ns}$. The energy measured with the hodoscope was required to be greater than $2 E_{\text {mip }}$, where $E_{\text {mip }}$ denotes the energy that the minimum ionizing particle deposits in a PS. The momentum of deuterons was calculated from the measured time delay.

A kinematic fit with six constraints was applied for the further event selection of the $\gamma d \rightarrow$ $\pi^{0} \pi^{0} d$ reaction. The required constraints were energy and three-momentum conservation between the initial and final states and two $\gamma \gamma$ invariant masses (the neutral-pion rest mass, $m_{\pi^{0}}$ ). Events in which the $\chi^{2}$ probability was higher than 0.4 were selected to prevent contamination from the background processes [11]. Here, sideband background subtraction was performed because accidental coincidence events exist between the photon-tagging counter and FOREST. 


\section{Total Cross Section}

Because the statistics were limited, the photon-tagging channels were divided into 16 groups, and the total cross section was obtained for each group. Fig. 1 1 shows the total cross section $\sigma$ for the $\gamma d \rightarrow \pi^{0} \pi^{0} d$ reaction as a function of the $\gamma d$ center-of-mass energy $W_{\gamma d}$. The total cross sections obtained for the 0.9 and $1.2 \mathrm{GeV}$ primary electrons were consistent within errors, and consistent with the existing theoretical calculation [12]. The cross section obtained for the $0.9 \mathrm{GeV}$ electrons was rather flat, and a clear resonance-like behavior was not observed in the excitation function for $W_{\gamma d}=2.38-2.61 \mathrm{GeV}$, where the $d^{*}(2380)$ dibaryon resonance observed at COSY was expected to appear. The $d^{*}(2380)$ contribution was estimated by fitting the function expressed as a sum of the expected $d^{*}(2380)$ contribution (a centroid of $M=2.37 \mathrm{GeV}$ and a width of $\Gamma=68 \mathrm{MeV}$ ) and background contribution (calculated cross section in Ref [12]). The total cross section was found to be $18.4 \pm 9.2 \mathrm{nb}$ at $W_{\gamma d}=2.37 \mathrm{GeV}$ and the corresponding upper limit was found to be $34 \mathrm{nb}$ (90\% confidence level). The details of the analysis for the $0.9-\mathrm{GeV}$ primary electrons including evaluation of the systematic uncertainties are described in Ref. [11].

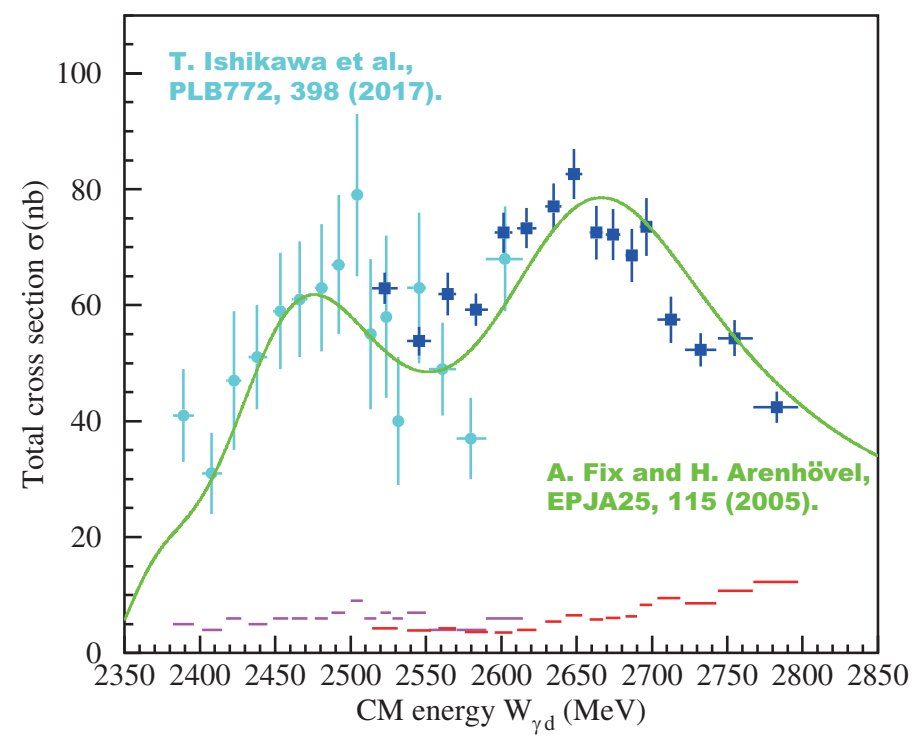

Figure 1: Total cross section $\sigma$ as a function of the $\gamma d$ center-of-mass energy $W_{\gamma d}$. The upper cyan and blue points show the obtained $\sigma$ for the 0.9 and $1.2 \mathrm{GeV}$ primary electron energies. The horizontal error of each point corresponds to the coverage of the incident photon energy, and the vertical error shows the statistical error of $\sigma$. The lower magenta and red histograms show the systematic error of $\sigma$. The solid green curve shows the calculated $\sigma$ given in Ref. [12].

\section{Differential Cross Section}

Because the statistics were limited, the photon-tagging channels were divided into 4 groups, and the $M_{\pi \pi}, M_{\pi d}$, and $\cos \theta_{d}$ differential cross sections were obtained for each group in the 1.2$\mathrm{GeV}$ electron mode. Fig. 2 shows the $M_{\pi \pi}, M_{\pi d}$, and $\cos \theta_{d}$ differential cross sections $d \sigma / d M_{\pi \pi}$, $d \sigma / d M_{\pi d}$, and $d \sigma / d \Omega_{d}$. The $d \sigma / d M_{\pi \pi}$ distribution did not show any enhancement at the low- 
and high-mass edges. The $d \sigma / d M_{\pi d}$ distribution showed a peak at approximately $2.15 \mathrm{GeV}$ with its reflection. The $d \sigma / d \Omega_{d}$ distribution showed a backward-peaking behavior although that for the lowest-energy tagging group was almost flat.
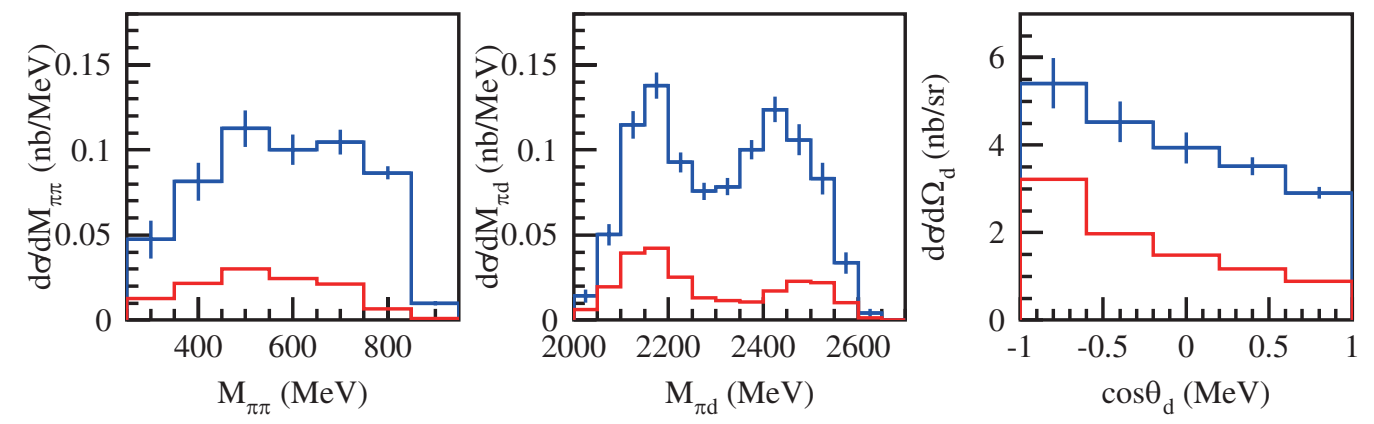

Figure 2: $M_{\pi \pi}, M_{\pi d}$, and $\cos \theta_{d}$ differential cross sections $d \sigma / d M_{\pi \pi}$ (left), $d \sigma / d M_{\pi d}$ (central), and $d \sigma / d \Omega_{d}$ (right). The cross sections for the highest-energy tagging group with $W_{\gamma d}=2749.7 \mathrm{MeV}$ (2703.6$2797.1 \mathrm{MeV}$ ) are plotted. The red histograms show the systematic uncertainties of the cross sections.

\section{Peak at $M_{\pi d} \sim 2.15 \mathrm{GeV}$}

Here, the peak observed in the $\pi d$ invariant mass distribution is discussed. At first, the mass and width were determined fitting a function, expressed as a sum of a Breit-Wigner peak, its reflection, and phase-space contributions, to the $d \sigma / d M_{\pi d}$ distribution shown in Fig. 3 (left). The obtained masses were $m=2160 \pm 3$ and $2146 \pm 3 \mathrm{MeV}$ for the highest and second tagging groups, respectively. The widths were $\Gamma=119 \pm 6$ and $110 \pm 3 \mathrm{MeV}$. The average values of the masses and widths were $m=2153 \pm 2 \mathrm{MeV}$ and $\Gamma=112 \pm 3 \mathrm{MeV}$. The mass of the peak is a little bit lower than the sum of the $N \Delta$ masses, and width is close to that for $\Delta$. The angular distribution was estimated for $\pi$ out of $\pi d$ system in its rest frame. Here, events in which $m_{\pi d}=2050-2250 \mathrm{MeV}$ were selected. Fig. 3 (right) shows the typical angular distribution of $\pi$ in the $\pi d$ rest frame. The $z$ axis was given as an opposite direction to the other $\pi$. The angular distribution shows a quadratic behavior convex downward, suggesting the angular momentum $L=1$ between $\pi d$.

\section{Summary}

The cross sections were measured for the $\gamma d \rightarrow \pi^{0} \pi^{0} d$ reaction at $E_{\gamma}=0.57--2.80 \mathrm{GeV}$. The total cross section was found to be $18.4 \pm 9.2 \mathrm{nb}$ at $W_{\gamma d}=2.37 \mathrm{GeV}$ for the $d^{*}(2380)$ dibaryon resonance. The measured excitation function was consistent with the existing theoretical calculation for this reaction. At high incident energies, $\pi^{0} d$ invariant-mass distributions show a peak with a mass of $m=2153 \pm 2 \mathrm{MeV}$, width of $\Gamma=112 \pm 3 \mathrm{MeV}$. The angular momentum of $L=1$ is suggested between $\pi^{0}$ and $d$. This peak can be attributed to a $N \Delta$ quasi-bound state. 

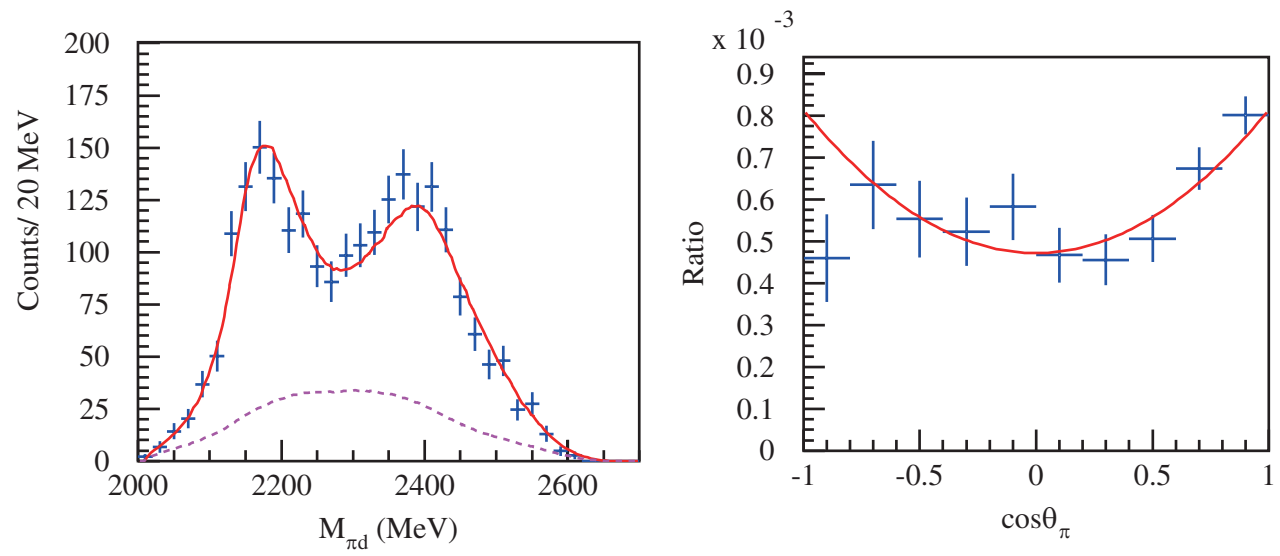

Figure 3: $M_{\pi d}$ differential cross section $d \sigma / d M_{\pi d}$ for the highest-energy tagging group (left). The solid curve shows the fitted function (see text), and the dotted corresponds to the phase space component. The angular distribution of $\pi$ in the $\pi d$ rest frame for the highest-energy tagging group (right). The events in which $M_{\pi d}=2050-2250 \mathrm{MeV}$ are selected. The $z$ axis is given as an opposite direction to the other $\pi$.

\section{Acknowledgments}

The authors would like to thank the accelerator staff at ELPH for their support during the FOREST experiment. This work was supported in part by JSPS KAKENHI Grants Nos.

$17340063,19002003,24244022,26400287$, and 16H02188.

\section{References}

[1] H. Clement, Prog. Part. Nucl. Phys. 93, 195 (2017).

[2] M. Bashkanov et al. (CELSIUS/WASA collaboration), Phys. Rev. Lett. 102, 052301 (2009).

[3] P. Adlarson et al. (WASA-at-COSY collaboration), Phys. Rev. Lett. 106, 242302 (2011); Phys. Lett. B 721, 229 (2013); Phys. Rev. C 88, 055208 (2013); Physics Lett. B 743, 325 (2015).

[4] H. Clement, M. Bashkanov and T. Skorodko, Physica Scripta T166, 014016 (2015).

[5] P. Adlarson et al. (WASA-at-COSY collaboration, SAID Data Analysis Center), Phys. Rev. Lett. 112, 202301 (2014); Phys. Rev. C 90, 035204 (2014).

[6] F.J. Dyson and N.-H. Xuong, Phys. Rev. Lett. 13, 815 (1964).

[7] T. Ishikawa et al., JPS Conf. Proc. 10, 031001 (2016).

[8] T. Ishikawa et al., Nucl. Instrum. Meth. A 622, 1 (2010); Nucl. Instrum. Meth. A 811, 124 (2016).

[9] F. Hinode et al., in: Proc. of 2005 Particle Accelerator Conference, 2458 (2005).

[10] T. Ishikawa et al., Nucl. Instrum. Meth. A 832, 108 (2016).

[11] T. Ishikawa et al., Phys. Lett. B 772, 398 (2017).

[12] A. Fix and H. Arenhövel, Euro. Phys. J. A 25, 115 (2005). 